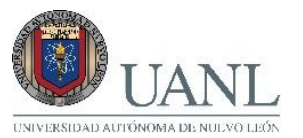

\title{
$\mathbf{R}$
}
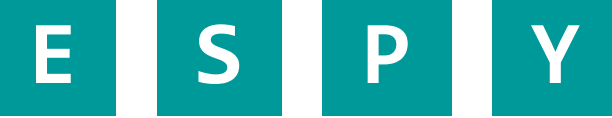

$\mathbf{N}$
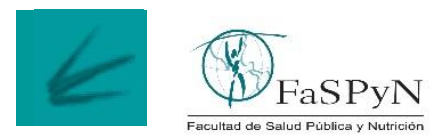

Revista Salud Pública y Nutrición

\section{EVALUACIÓN NUTRICIONAL EN ESTUDIANTES DE UNA UNIVERSIDAD PÚBLICA.}

\author{
NUTRITIONAL ASSESSMENT IN STUDENTS OF A PUBLIC UNIVERSITY.
}

Mallqui More Juan Edwin ${ }^{1}$, Leon Toledo Liz Emyli ${ }^{1}$, Reyes Narváez Silvia Elizabet ${ }^{2}$.

1 Universidad Nacional de Barranca, Perú. 2 Universidad Nacional Santiago Antúnez de Mayolo, Perú.

Citation: Mallqui More J.E., Leon Toledo L.E., Reyes Narváez S.E. (2020).

Evaluación nutricional en estudiantes de una universidad pública. Revista

Salud Pública y Nutrición, 19 (4), 10-15.

Editor: Esteban G. Ramos Peña, Dr. CS., Universidad Autónoma de Nuevo León, Facultad de Salud Pública y Nutrición, Monterrey Nuevo León, México. Copyright: (C)2020 Mallqui More J.E., et al. This is an open-access article distributed under the terms of Creative Commons Attribution License [CC BY 4.0], which permits unrestricted use, distribution, and reproduction in any medium, provided the original author and source are credited.

Competing interests: The authors have declared that no competing interests exist.

DOI: https://doi.org/10.29105/respyn19.4-2

Recibido: 03 de julio 2020;

Aceptado: 05 de octubre 2020

Email: reynaelizabet26@hotmail.com 


\title{
EVALUACIÓN NUTRICIONAL EN ESTUDIANTES DE UNA UNIVERSIDAD PÚBLICA.
}

\author{
Mallqui More Juan Edwin ${ }^{1}$, Leon Toledo Liz Emyli ${ }^{1}$, Reyes Narváez Silvia Elizabet ${ }^{2}$.
}

1 Universidad Nacional de Barranca, Perú. 2 Universidad Nacional Santiago Antúnez de Mayolo, Perú.

\section{RESUMEN}

Introducción. Los hábitos alimentarios de los estudiantes universitarios varían por diversos factores que afectan su estado nutricional ocasionando la prevalencia de obesidad y sobrepeso, siendo estas, las que aumentan el riesgo de padecer enfermedades no trasmisibles (ENT). Objetivo: Determinar la relación entre los hábitos alimenticios y el estado nutricional de los estudiantes de una universidad pública en Barranca. Material y Método: Investigación descriptiva correlacional de diseño no experimental, en una muestra de 261 estudiantes de 7 escuelas profesionales seleccionados a través un muestreo aleatorio estratificado. La recolección de datos se realizó con una encuesta de hábitos alimenticios, clasificándolo en categoría Adecuado (15-20 puntos) e Inadecuado (0-14 puntos). La evaluación nutricional se obtuvo a través del índice de masa corporal (IMC), clasificando en delgadez, normal, sobrepeso y obesidad. Resultados: $80.4 \%$ de estudiantes universitarios presenta hábitos alimenticios inadecuados y $52.9 \%$ sobrepeso. Por escuelas profesionales se observa $42.9 \%$ con obesidad tipo II en Ingeniería agrónoma, $24.2 \%$ con obesidad tipo I en Enfermería y $20.3 \%$ con sobrepeso en Contabilidad y finanzas. Conclusiones: La prueba de hipótesis evidencia que existe relación significativa entre los hábitos alimenticios y el estado nutricional $(p=0.00)$, y el estado nutricional no guarda relación con la escuela profesional $(p=0.11)$.

Palabras Clave: Evaluación nutricional, conducta alimentaria, índice de masa corporal (IMC).

\section{ABSTRACT}

Introduction: The eating habits of university students vary due to various factors that affect their nutritional status, causing the prevalence of obesity and overweight, these being the ones that increase the risk of suffering from non-communicable diseases (ENT). Objective: To determine the relationship between eating habits and nutritional status of students at a public university in Barranca. Material and method: Descriptive correlational research of non-experimental design, in a sample of 261 students from 7 professional schools selected through stratified random sampling. Data collection was carried out with a survey of eating habits, classifying it in the Adequate (15-20 points) and Inadequate (0-14 points) categories. The nutritional evaluation was obtained through the body mass index (BMI), classifying as thin, normal, overweight and obesity. Results: $80.4 \%$ of university students present inadequate eating habits and $52.9 \%$ overweight. By professional schools, $42.9 \%$ were observed with type II obesity in Agronomic Engineering, $24.2 \%$ with type I obesity in Nursing and $20.3 \%$ with overweight in Accounting and finance. Conclusions: The hypothesis test shows that there is a significant relationship between eating habits and nutritional status $(p=0.00)$, and nutritional status is not related to professional school $(p=0.11)$.

Key words: Nutritional evaluation, eating behavior, body mass index (BMI). 


\section{Introducción}

La etapa universitaria suele coincidir con el paso de la adolescencia a la adultez y es en ese momento que los estudiantes se responsabilizan por primera vez de su alimentación. Este grupo etario se deben considerar como un grupo de intervención para mejorar sus hábitos alimentarios el cual ha empeorado en los últimos años colocándolo en alto riesgo de padecer enfermedades crónicas no trasmisibles (ENT) a corto, mediano y largo plazo (Concha, Gonzales, Puñuñuri y Valenzuela, 2019).

Esta etapa importante de su vida es especial para la toma de decisiones respecto a su salud, asimismo, para la promoción de estilos de vida saludable (Riveros y Apolaya, 2020). Los jóvenes universitarios se enfrentan a cambios en su estilo de vida compatibilizando los estudios con la actividad física, social y hábitos alimentarios. Además, los cánones de belleza que fomentan la delgadez en las mujeres y la musculatura en los varones influyen en su autoimagen corporal que pueden derivar en conductas alimentarias nocivas para su salud (Zulet y col, 2018).

A nivel mundial se estima que 1900 millones de adultos mayores de 18 años tiene sobrepeso y 650 millones obesidad (OMS, 2020). En este contexto el Perú evidencia $42.4 \%$ de jóvenes y $23.9 \%$ de adolescentes con sobrepeso y obesidad; asimismo, una vez a la semana el $29 \%$ de personas consumen comida chatarra y $87,1 \%$ frituras; mientras que $20.2 \%$ consume un exceso de sal (INS, 2019).

El sobrepeso y obesidad es un problema social y sanitario de gran magnitud (Zulet y col, 2018), que se observa en diversas regiones del país relacionado con la ingesta alimentaria y situaciones del núcleo familiar (Alvines y col. 2019). La obesidad se asocia a carencias nutricionales en los primeros años de vida, a los hábitos alimentarios, las condiciones socioculturales, la actividad física y al consumo de bebidas azucaradas (Cárdenas y col. 2019). De igual modo la elevada prevalencia posiblemente este asociado al sedentarismo y al consumo de alimentos hipercalóricos y ultraprocesados, Calderón y col. (2018), encontró que los jóvenes tienen preferencias alimentarias por las pastas el 79,58\%, 65.65\% por los snacks y el $63.68 \%$ la carne procesada; además no gustan de las verduras y el $22.48 \%$ no le gusta ningún tipo de verdura.
Los universitarios son conscientes que una alimentación saludable es beneficiosa para su salud física y mental, sin embargo, es más costosa y les toma tiempo la compra y preparación, además de los horarios y restricciones económicas propias de su etapa (Sánchez y col. 2019).

La alimentación de los universitarios mexicanos es poco saludable. Un estudio en la Universidad de Guadalajara a estudiantes del área de salud y otras áreas los jóvenes reconocieron que una alimentación saludable les da bienestar físico y mental. En lo físico refieren que les da más energía, mejor funcionamiento de los órganos y les previene de ENT. En relación a lo mental se sienten realizados, con mayor autoestima y mejor rendimiento académico. No obstante, ellos atribuyen el rol esencial de la madre en las decisiones de elección e ingesta de alimentos (Sánchez y col. 2019).

La familia constituye un pilar fundamental en la alimentación de sus integrantes, así lo demostró Alvines y col. (2019), en un estudio realizado en estudiantes universitarios del interior del país y de la zona norte de Lima cuyo estilo de vida se vio reflejado en la prevalencia de sobrepeso y obesidad (21.6\% y $8.9 \%$ ) y el $21 \%$ presento alto riesgo de ENT. De igual modo se encontró 50\% de sobrepeso y obesidad y riesgo de enfermedad cardiovascular relacionada a la malnutrición en estudiantes de medicina humana en Lima-Perú (Riveros y Apolaya, 2020).

Supuestamente a mayor conocimiento en temas de salud mejor serán los hábitos alimentarios, sin embargo, no se encontró diferencias significativas entre el comportamiento alimentarios de los estudiantes de Ciencias de la Salud en relación a las otras carreras profesionales, lo que indica que los conocimientos que tienen sobre nutrición no lo ponen en práctica. Pues los jóvenes deciden que alimentos consumen influenciados por los diversos factores como la presión social, la disponibilidad de dinero, la mayor independencia o la oferta de comidas rápidas. De allí que se debe promover los buenos hábitos alimentarios para alcanzar un adecuado estado nutricional y la manera más conveniente, es incorporando una gran variedad de alimentos en la dieta diaria (Muñoz y col. 2017). 
Por consiguiente, el objetivo de este estudio es conocer el estado nutricional de los estudiantes de una universidad pública, lo que nos permitirá elaborar propuestas de intervención para fortalecer los estilos de vida saludable relacionado a la alimentación y con ello contribuir a mejorar los índices de prevalencia de obesidad y sobrepeso en este grupo poblacional.

\section{Material y Método}

El presente estudio es una investigación básica de diseño no experimental realizada en el año 2019. La muestra fue de 261 estudiantes universitarios de las escuelas profesionales de Ingeniería civil, Ingeniera agrónoma, Ingeniería de industrias alimentarias, Enfermería, Obstetricia, Contabilidad y Derecho, seleccionados a través un muestreo aleatorio estratificado. Los criterios de inclusión se consideró que estén matriculados en el semestre 2019-II, que cursen estudios del I al VIII ciclo, de ambos sexos y que acepten participar voluntariamente.

El diagnóstico nutricional se realizó a través de la medición antropométrica para obtener el IMC, clasificando según los criterios de la Organización Mundial de la Salud (OMS) en: Delgadez tipo III (< 16), Delgadez tipo II (16 a < 17), Delgadez tipo I (17 a < 18.5), Normal (18.5 a < 25), sobrepeso $(25 \mathrm{a}<$ 30), Obesidad I (30 a < 35), Obesidad II (35 a < 40) y Obesidad III ( $\geq$ a 40) (OMS, 1995). Para la toma de datos se consideró la Guía técnica para la valoración nutricional antropométrica de la persona adulta del Misterio de Salud (Minsa), el peso se midió con una balanza de plataforma y la talla se utilizó el tallímetro (MINSA, 2012).

Para medir la segunda variable se utilizó el cuestionario Conociendo tus hábitos alimentarios, elaborado por los autores basada en la Norma técnica de alimentación para la Población Peruana (2018), constó de 20 preguntas cerradas, con una valoración de 1 punto para la respuesta correcta, clasificando en hábitos alimenticios adecuados (15-20 puntos) e inadecuados (0-14 puntos). Este instrumento tuvo un coeficiente de validez de contenido (CVC) de 0,94 y de confiabilidad 0,86 según la prueba estadística de Kuder - Richardson (KR -20). El análisis de los datos se realizó con el SPSS versión 20 y se presentó los resultados en tablas de contingencia. Se consideró el nivel de confianza de $95 \%$ y se aplicó la prueba de Chi cuadrado para la relación de variables. Se aplicó los principios éticos y bioéticos de la investigación aplicables en cualquier tipo de investigación y se consideró el anonimato y privacidad, de igual modo los jóvenes que aceptaron participar firmaron el consentimiento informado. Previamente este consentimiento fue aprobado por el Comité de Ética de la Universidad.

\section{Resultados}

El estudio estuvo conformado por 261 estudiantes universitarios, de este grupo 94.3\%(246) de ellos comprende el grupo de edad de 18 a 29 años, $52.9 \%(138)$ son varones y $47.1 \%$ (123) mujeres; $71.6 \%(187)$ proceden del área urbana y el 28.4\%(74) del área rural, $93.9 \%$ (245) son de estado civil soltero; $60.9 \%(159)$ no trabajan y el $26.4 \%(69)$ tiene un trabajo independiente (ver tabla 1).

\begin{tabular}{|c|c|c|c|}
\hline & & $\mathrm{N}^{\circ}$ & $\%$ \\
\hline \multirow{3}{*}{ Grupo edad (años) } & $<18$ & 4 & 1,5 \\
\hline & $18-29$ & 246 & 94,3 \\
\hline & $>30$ & 11 & 4,2 \\
\hline \multirow{2}{*}{ Sexo } & Hombre & 138 & 52,9 \\
\hline & Mujer & 123 & 47,1 \\
\hline \multirow{2}{*}{ Procedencia } & Urbano & 187 & 71,6 \\
\hline & Rural & 74 & 28,4 \\
\hline \multirow{2}{*}{ Estado civil } & Soltero & 245 & 93,8 \\
\hline & Casado & 2 & 0,8 \\
\hline \multirow{4}{*}{ Ocupación } & Otros & 14 & 5,4 \\
\hline & No trabaja & 175 & 67 \\
\hline & Trabajador independiente & 69 & 26,4 \\
\hline & Trabajador dependiente & 17 & 6,6 \\
\hline
\end{tabular}

Fuente: Elaboración Propia

El $80.5 \%$ (210) de los estudiantes tienen hábitos alimenticios inadecuados y el 19.5\% (51) hábitos alimenticios adecuados. Por consiguiente, se evidencia que existen hábitos alimenticos inadecuados en los estudiantes universitarios (ver tabla 2).

Tabla 2. Hábitos alimenticios de los estudiantes universitarios

\begin{tabular}{llcc}
\hline & & $\mathrm{N}^{\circ}$ & $\%$ \\
\hline \multirow{2}{*}{ Hábitos } & Adecuado & 51 & 19.5 \\
alimenticios & Inadecuado & 210 & 80.5 \\
& Total & 261 & 100 \\
\hline
\end{tabular}

Fuente: Elaboración Propia 
En relación al diagnóstico nutricional se puede aprecia que el 52.9\% (138) alcanzó un diagnóstico nutricional de sobrepeso. Por consiguiente, se afirma que el índice de masa corporal es anormal en la mayoría de los estudiantes (ver tabla 3).

Tabla 3. Diagnóstico nutricional de estudiantes universitarios

\begin{tabular}{llcc}
\hline & & $\mathrm{N}^{\circ}$ & $\%$ \\
\hline & Delgadez tipo I & 7 & 2.7 \\
& Normal & 76 & 29.1 \\
Diagnóstico & Sobrepeso & 138 & 52.9 \\
nutricional & Obesidad tipo I & 33 & 12.6 \\
& Obesidad tipo II & 7 & 2.7 \\
& Total & 261 & 100 \\
\hline
\end{tabular}

Fuente: Elaboración Propia

En relación al diagnóstico nutricional según hábitos alimenticios se puede observar que los estudiantes universitarios con hábitos alimenticios inadecuados evidencian malnutrición por exceso y carencia, como el sobrepeso, obesidad y delgadez; y los que tienen hábitos alimenticios adecuados reportan un IMC normal, por lo tanto, existe relación significativa entre los hábitos alimenticios y el estado nutricional $(\mathrm{p}=0.00)($ ver tabla 4$)$.

\begin{tabular}{|c|c|c|c|c|c|c|c|c|c|c|c|c|}
\hline \multirow{4}{*}{$\begin{array}{c}\text { Hábitos } \\
\text { Alimenticios } \\
\text { Inadecuado }\end{array}$} & \multicolumn{12}{|c|}{ Diagnóstico nutricional } \\
\hline & \multirow{2}{*}{\multicolumn{2}{|c|}{$\begin{array}{c}\text { Delgadez I } \\
\mathrm{N}^{\circ}(\%)\end{array}$}} & \multirow{2}{*}{\multicolumn{2}{|c|}{$\begin{array}{l}\text { Normal } \\
\mathrm{N}^{\circ}(\%)\end{array}$}} & \multirow{2}{*}{\multicolumn{2}{|c|}{$\begin{array}{c}\text { Sobrepeso } \\
\mathrm{N}^{\circ}(\%)\end{array}$}} & \multirow{2}{*}{\multicolumn{2}{|c|}{$\begin{array}{c}\text { Obesidad I } \\
\mathrm{N}^{\circ}(\%)\end{array}$}} & \multirow{2}{*}{\multicolumn{2}{|c|}{$\begin{array}{c}\text { Obesidad II } \\
\mathrm{N}^{\circ}(\%)\end{array}$}} & \multicolumn{2}{|c|}{ Total } \\
\hline & & & & & & & & & & & $\mathrm{N}^{\circ}$ & (\%) \\
\hline & 7 & 2.7 & 26 & 9.9 & 137 & 52.5 & 33 & 12.6 & 7 & 2.7 & 210 & 80.4 \\
\hline Adecuado & 0 & 0 & 50 & 19.2 & 1 & 0.4 & 0 & 0 & 0 & 0 & 51 & 19.6 \\
\hline Total & 7 & 2.7 & 76 & 29.1 & 138 & 52.9 & 33 & 12.6 & 7 & 2.7 & 261 & 100 \\
\hline
\end{tabular}

En el diagnóstico nutricional por escuelas profesionales se observa que $42.9 \%$ de los estudiantes de Ingeniería agrónoma $42.9 \%$ presenta obesidad tipo II, en Enfermería 24.2\% tiene obesidad tipo I y en Contabilidad y finanzas $20.3 \%$ tiene sobrepeso. Según la prueba de Chi cuadrado el estado nutricional no tiene relación con la escuela profesional $(\mathrm{p}=0.11)$ (ver tabla 5).

\begin{tabular}{|c|c|c|c|c|c|c|c|c|c|c|}
\hline \multirow{3}{*}{$\begin{array}{c}\text { Escuela } \\
\text { Profesional }\end{array}$} & \multicolumn{10}{|c|}{ Diagnóstico nutricional } \\
\hline & \multicolumn{2}{|c|}{$\begin{array}{c}\text { Sobrepeso } \\
\mathrm{N}^{\circ}(\%)\end{array}$} & \multicolumn{2}{|c|}{$\begin{array}{c}\text { Normal } \\
\mathrm{N}^{\circ}(\%)\end{array}$} & \multicolumn{2}{|c|}{$\begin{array}{c}\text { Obesidad I } \\
\mathrm{N}^{\circ}(\%)\end{array}$} & \multicolumn{2}{|c|}{$\begin{array}{c}\text { Obesidad II } \\
\mathrm{N}^{\circ}(\%)\end{array}$} & \multicolumn{2}{|c|}{$\begin{array}{c}\text { Delgadez I } \\
\mathrm{N}^{\circ}(\%)\end{array}$} \\
\hline & 23 & 16.7 & 18 & 23.7 & 7 & 21.2 & 1 & 14.3 & 1 & 14.3 \\
\hline $\begin{array}{l}\text { Contabilidad y } \\
\text { Finanzas }\end{array}$ & 28 & 20.3 & 11 & 14.5 & 2 & 6.1 & 0 & 0 & 1 & 14.3 \\
\hline $\begin{array}{l}\text { Derecho y } \\
\text { Ciencia Política }\end{array}$ & 13 & 9.4 & 14 & 18.4 & 6 & 18.2 & 1 & 14.3 & 2 & 28.5 \\
\hline Enfermería & 20 & 14.5 & 5 & 6.6 & 8 & 24.2 & 1 & 14.3 & 0 & 0 \\
\hline Obstetricia & 19 & 13.8 & 5 & 6.6 & 7 & 21.2 & 0 & 0 & 0 & 0 \\
\hline Ing. Agrónoma & 19 & 13.8 & 9 & 11.8 & 1 & 3 & 3 & 42.8 & 2 & 28.5 \\
\hline $\begin{array}{l}\text { Ing. Industrias } \\
\text { Alimentarias }\end{array}$ & 16 & 11.5 & 14 & 18.4 & 2 & 6.1 & 1 & 14.3 & 1 & 14.3 \\
\hline Total & 138 & 100 & 76 & 100 & 33 & 100 & 7 & 100 & 7 & 100 \\
\hline
\end{tabular}

\section{Discusión}

Según la revista de psicología (Becerra, 2016), la vida universitaria es una etapa de crecimiento individual, donde la persona se desarrolla física y cognitivamente, además la persona adquiere conductas relacionadas a la salud sean o no beneficiosas. Sin embargo, los jóvenes asumen el autocuidado de su salud para mantener un estilo de vida saludable.

Teniendo los diagnósticos nutricionales con mayor incidencia en sobrepeso y obesidad en estudiantes de una universidad pública, se encontró una relación significativa entre los hábitos alimenticios y el diagnóstico nutricional. De un total de $100 \%$ el $80.5 \%$ presentó hábitos alimenticios inadecuados repercutiendo en el $52.9 \%$ de sobrepeso en estudiantes universitarios. Esta relación se contrasta con la realidad nacional, que a pesar de la existencia de lineamientos de políticas de salud que tienen como objetivo disminuir enfermedades no trasmisibles y fomentar estilos de vida saludable, aún se sigue registrando este problema en el sector de salud pública. Esta situación no solo afecta a la población que se encuentra en etapa adolescente sino también está incluido un grupo etario muy vulnerable a cambios que alteran su estilo de vida como es la juventud.

Los resultados obtenidos de sobrepeso y obesidad fueron de $68.2 \%$, siendo una cifra alarmante para la salud pública. Según el estudio los estudios esta población presenta alto riesgo cardiovascular (Riveros y Apolaya, 2020). Estos resultados discrepan al estudio sobre hábito y calidad nutricional del desayuno en estudiantes de nutrición donde el $69.4 \%$ presento resultado normal (Herrera, 
Celoira y Asaduroglu, 2017). También al estudio estilo de vida saludable en estudiantes de enfermería donde se evidenció que el $95 \%$ presento un índice de masa corporal normal (Morales, Gómez, García, Chaparro y Carreño, 2018).

La obesidad y sobrepeso es más prevalente en la etapa vida joven, el cual puede generar un impacto decisivo en la vida adulta. En este proceso el profesional de enfermería urge la necesidad de realizar promoción y prevención a fin de garantizar un buen estado de salud e identificando los factores de riesgo en forma oportuna (MINSA, 2018).

En relación al diagnóstico nutricional y hábitos alimenticios según escuelas profesionales, se evidencio que el $42.8 \%$ de los estudiantes que pertenecen a la escuela profesional de ingeniería agrónoma presentan obesidad tipo II. Estos resultados se asemejan al estudio conocimientos sobre alimentación saludable, donde se demostró que el $63.2 \%$ de los estudiantes de dicha escuela profesional presentan un bajo nivel de conocimientos sobre alimentación saludable. (Reyes y Oyola, 2020).

Si los estudiantes continúan con los hábitos alimentarios inadecuados, en el futuro pueden tener ENT generando un costo económico personal, familiar y al estado. El estudiante universitario debe tomar conciencia de su cuidado y adoptar buenos hábitos alimenticios (Botello, Villanueva, Ruiz y Gallegos, 2018). Callisaya (2016) citado por Aco (2018), refiere que los horarios de clase muchas veces impiden que los estudiantes ingieran sus alimentos en la hora adecuada, y en ocasiones suspendan los platos esenciales de la alimentación. Los jóvenes requieren alimentarse para un buen desempeño académico, caso contrario puede generar agotamiento y distracción.

De acuerdo a la evidencia científica la etapa universitaria tiene gran relevancia en la adopción de hábitos alimentarios en la población joven (Olea, Celis, Granfeldt, 2019). Además, está demostrado que los hábitos alimenticios se relacionan con el IMC, donde la universidad interviene de forma directa e indirecta en sus hábitos alimenticios. Por tal razón la universidad dentro de la formación integral debe promover estilos de vida saludable, dentro de ellos la buena alimentación.

\section{Conclusiones}

Existe relación significativa entre los hábitos alimenticios y el estado nutricional de los estudiantes de una universidad pública los mismos que presentan riesgo de padecer ENT al tener hábitos alimenticios inadecuados y sobrepeso. Asimismo, el estado nutricional no guarda relación con la escuela profesional. Se sugiere realizar programas de intervención para este grupo poblacional con el propósito de fortalecer sus prácticas de alimentación que eviten la omisión de los principales platos de comida y el excesivo consumo de grasas saturadas, carbohidratos y comidas rápidas.

\section{Bibliografía}

Aco, C. (2018). Hábitos alimenticios de los estudiantes ingresantes a la Universidad Andina del Cusco 2018. Yachay, 7 (1): 334-338. https://doi.org/10.36881/yachay.v7i01.81

Alvines, D., Suárez, C., Matta, H., Carcausto, W. (2019). Obesidad y sobrepeso según tipología familiar de los estudiantes de una universidad privada de Lima. Health Care \& Global Health, 3(1):17-22.

http://dx.doi.org/10.22258/hgh.2019.31.50

Becerra, S. (2016). Descripción de las conductas de salud en un grupo de estudiantes universitarios de Lima. Revista de Psicología, 34(2), 1-22. http://dx.doi.org/10.18800/psico.201602.001

Botello, M., Villanueva, L., Ruiz, V., Gallegos, T. (2018). Alimentación y estilos de vida saludables percibidos en estudiantes universitarios del área de la salud, de tres licenciaturas, México. Revista Lux Médica, 39, 55-62. https://doi.org/10.33064/391m20181334

Calderón, A., Marrodán, M., Villarino, A. y Martínez, J. (2019). Valoración del estado nutricional y de hábitos y preferencias alimentarias en una población infanto-juvenil (7 a 16 años) de la Comunidad de Madrid. Nutr Hosp, 36(2):394-404. http://dx.doi.org/10.20960/nh.2244

Cárdenas, D., Calvo, V., Flores, S., Sepúlveda, D. y Manjarres, L. (2019). Consumo de bebidas azucaradas y con azúcar añadida y su asociación con indicadores antropométricos en jóvenes de 
Medellín (Colombia). Nutr Hosp, 36(6):13461353. http://dx.doi.org/10.20960/nh.02671

Concha, C., González, G., Piñuñuri, R., Valenzuela, C. (2019). Relación entre tiempos de alimentación, composición nutricional del desayuno y estado nutricional en estudiantes universitarios de Valparaíso, Chile. Rev Chil Nutr, 46(4), 400-408. http://dx.doi.org/10.4067/S0717-

75182019000400400

Fraile, P., Lizancos, A., Melero, A., González, C., Monereo, S., Calvo, S. (2020). Relación de la composición corporal medida por DEXA con el estilo de vida y la satisfacción con la imagen corporal en estudiantes universitarios. Nutr Hosp, $36(4)$, 919-925.

http://dx.doi.org/10.20960/nh.2103

Herrera, G., Celoira, M., Asaduroglu, A. (2017). Hábito y calidad nutricional del desayuno en estudiantes que cursan la Carrera Licenciatura en Nutrición, Universidad Nacional de Córdova, Argentina. Rev. Esp. Nutr. Comunitaria, 23(2), 14-21. http://dx.doi.org/10.14642/RENC.2017.23.sup2. 5173

Instituto Nacional de Salud [INS] (2019). Cerca del $70 \%$ de adultos peruanos padecen de obesidad y sobrepeso. Lima-Perú. https://web.ins.gob.pe/es/prensa/noticia/cercadel-70-de-adultos-peruanos-padecen-deobesidad-y-sobrepeso

Ministerio de Salud [MINSA] (2018). Norma Técnica-Guías Alimentarias para la Población Peruana. Perú. 2018. N: 1353-2018 / MINSA. Lima-Perú.

Ministerio de Salud [MINSA] (2018). Norma Técnica-Orientaciones para la atención integral de salud en la etapa de vida Joven. $\mathrm{N}^{\circ}$ : 456-2016 / MINSA. Lima-Perú.

Morales, M., Gómez, V., García, C., Chaparro, L., Carreño, S. (2018). Estilo de vida saludable en estudiantes de enfermería del Estado de México. Rev. Colomb. Enferm. 4(16), 14-24. http://dx.doi.org/10.18270/rce.v16i13.2300
Muñoz de Mier, G., Lozano, M., Romero, C., Pérez de Diego, J. y Veiga, P. (2017). Evaluación del consumo de alimentos de una población de estudiantes universitarios y su relación con el perfil académico. Nutr Hosp, 34(1), 134-143. http://dx.doi.org/10.20960/nh.989

Olea, M., Celis, C., Granfeldt, G. (2019). Efecto de una intervención dieto terapéutica con diferentes aportes de calcio dietario sobre el estado nutricional en estudiantes universitarias con sobrepeso. Rev Chil Nutr, 46(4): 460-468. http://dx.doi.org/10.4067/S071775182019000400460

Organización Mundial de la Salud [OMS] (2020). Obesidad y sobrepeso. Nota descriptiva. Ginebra. https://www.who.int/es/newsroom/fact-sheets/detail/obesity-and-overweight

Organización Mundial de la Salud [OMS] (1995). El estado físico: uso e interpretación de la Antropometría. Informe del Comité de Expertos de la OMS. Serie de Informes técnicos 854. Ginebra. Disponible en: http://whqlibdoc.who.int/trs/WHO_TRS_854_co ntents_spa.pdf

Reyes, S., y Oyola, M. (2020). Conocimientos sobre alimentación saludable en estudiantes de una universidad pública. Rev Chil Nutr, 47(1), 67-72. http://dx.doi.org/10.4067/S071775182020000100067

Riveros, S., y Apolaya, M. (2020). Características clínicas y epidemiológicas del estado nutricional en ingresantes a la facultad de medicina humana de una universidad privada. Acta Med Perú, $37(1), 27-33$.

http://dx.doi.org/10.35663/amp.2020.371.965

Sánchez, J., Martínez, A., Nazar, G., Mosso, C., Del Muro, L. (2019). Creencias alimentarias en estudiantes universitarios mexicanos: Una aproximación cualitativa. Rev Chil Nutr, 46(6): 727-734. http://dx.doi.org/10.4067/S071775182019000600727 